\title{
Régulation des stations de pompage et des réseaux sous pression
}

\author{
Christian Coite
}

Bas-Rhône-Languedoc

\section{Introduction}

La régulation des stations de pompage et des réseaux d'irrigation sous pression présente un certain nombre de caractéristiques partiellement ou en totalité conformes aux principes retenus à $\mathrm{BRL}$ :

- réseaux «à la demande » c'est-à-dire que l'eau doit être disponible à toute heure et pour quelque débit que ce soit (pour certains ouvrages cette règle s'applique aussi en hiver) ;

— pas de réserve de sécurité (en cas de coupure d'énergie, arrêt de la fourniture d'eau) ;

— produits (eau agricole) de faible valeur ajoutée, d'où nécessité d'une gestion très serrée du coût actualisé investissement et fonctionnement.

On ne peut étudier en détail la régulation d'un tel système de pompage sans se poser la question pourquoi, et pour qui, faire de la régulation.

\section{Pourquoi?}

En général, les raisons qui poussent à utiliser une régulation sont les suivantes :

- économie d'énergie : Connaissant la courbe de demande $\mathrm{H}=\mathrm{f}(\mathrm{Q})$ on peut, par une régulation, faire correspondre plus ou moins la colline des rendements optimaux avec cette dernière (voir schémas 1, 2 et 3).

- économie de personnel: Plus les dispositifs de contrôle (débit, pression, vannes pression amont ou aval, vitesse de rotation, nature et vitesse d'ouverture d'une vanne au refoulement, automates) vont être nombreux, plus l'installation va être autonome et s'affranchir d'une présence, même temporaire (voir schéma 4). Ceci permet de réduire les frais d'exploitation. En contrepartie, l'automatisation peut conduire à des dépenses d'entretien et maintenance plus élevées.

- progressivité des investissements: La régulation, lorsqu'elle permet une variation progressive dans le fonctionnement de l'installation ou au cours du temps, permet aussi une progression dans les investissements périphériques (par exemple de protection) à l'intérieur ou à l'ex- térieur de la station de pompage (conduite de refoulement...).

- amélioration de la sécurité : Ce critère souvent retenu au bénéfice de la régulation doit cependant être nuancé si l'installation doit continuer à fonctionner en cas de panne celle-ci.

\section{Pour qui ?}

On pensera ici beaucoup moins au maître d'ouvrage qu'à l'exploitant lui-même. Ce dernier pouvant avoir un niveau de compétences situé entre les deux extrêmes qui sont :

- le petit syndicat d'agriculteurs "fermé », l'un d'entre eux étant logé à côté de la station de pompage ;

- le grand périmètre irrigué "à la demande" faisant lui-même partie d'un vaste réseau dont le fonctionnement est identique avec une structure centrale d'exploitation.

\section{Les divers types de régulation}

Avant d'étudier les implications économiques de telle ou telle régulation, il importe, bien qu'ils soient largement connus aujourd'hui, de rappeler les divers modes de régulation à la disposition de l'ingénieur conseil.

Le problème à régler est d'assurer le fonctionnement de la station sur la totalité de la plage des débits du réseau en adaptant le nombre de groupes en fonctionnement.

\section{Solution 1: Ne rien faire}

On oublie souvent que la première possibilité est tout simplement de ne rien prévoir, la mise en marche du ou des groupe(s) se faisant manuellement par un responsable désigné à cet effet. La seule précaution étant ici de se prémunir contre les risques de dépression dus à une coupure d'énergie par un système de by-pass avec clapet et ventouse et de bien informer l'exploitant sur les limites de fonctionnement de la pompe (en matière de débit) et du réseau (en matière de pression) (voir schémas 5 et 5 bis). 


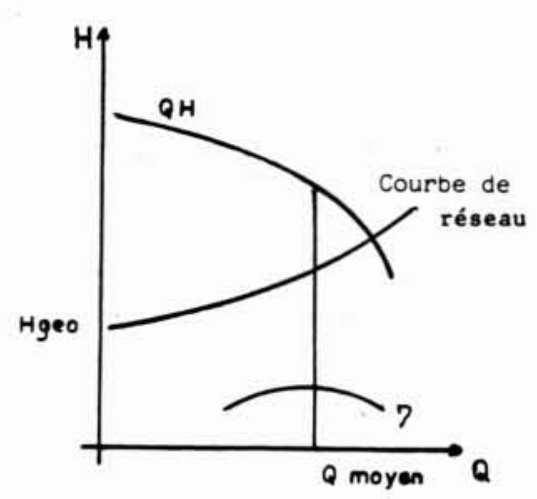

1. Une pompe à vitesse fixe.

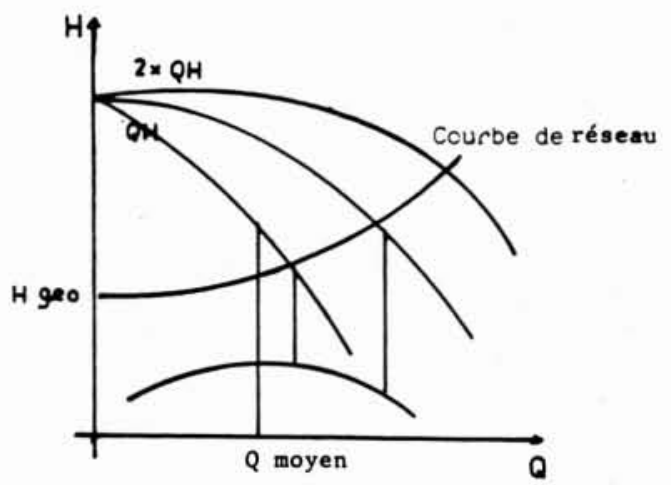

2. Deux pompes à vitesse fixe.

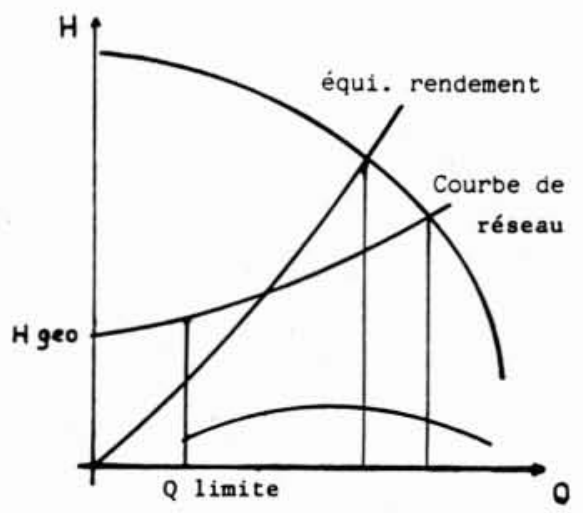

3. Une pompe à vitesse variable.

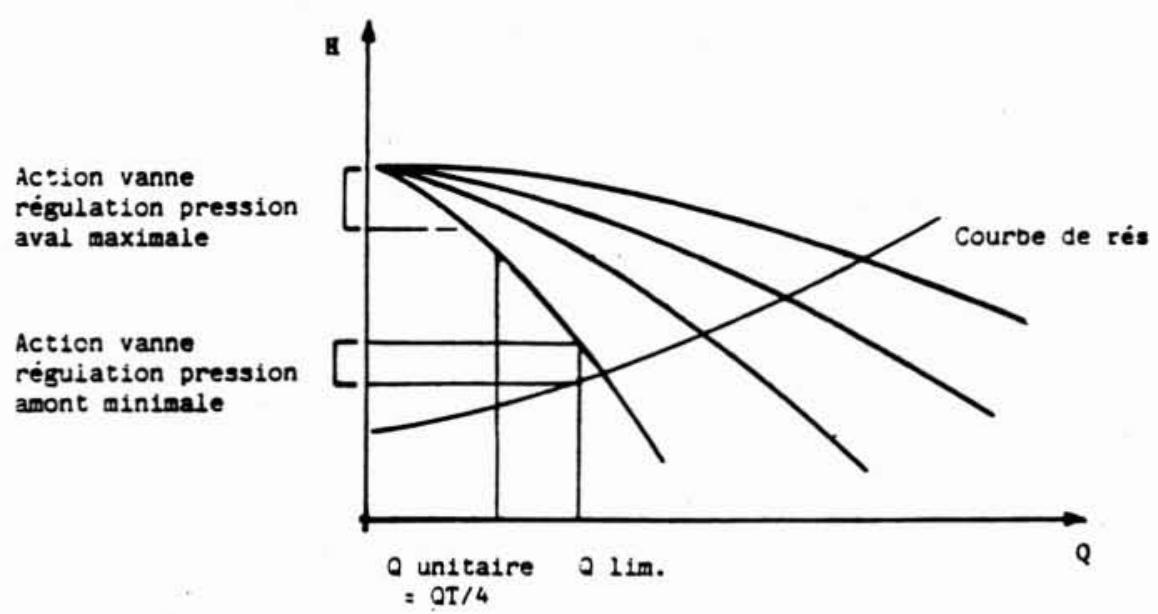

4. Régulation par vannes aval et (ou) amont.

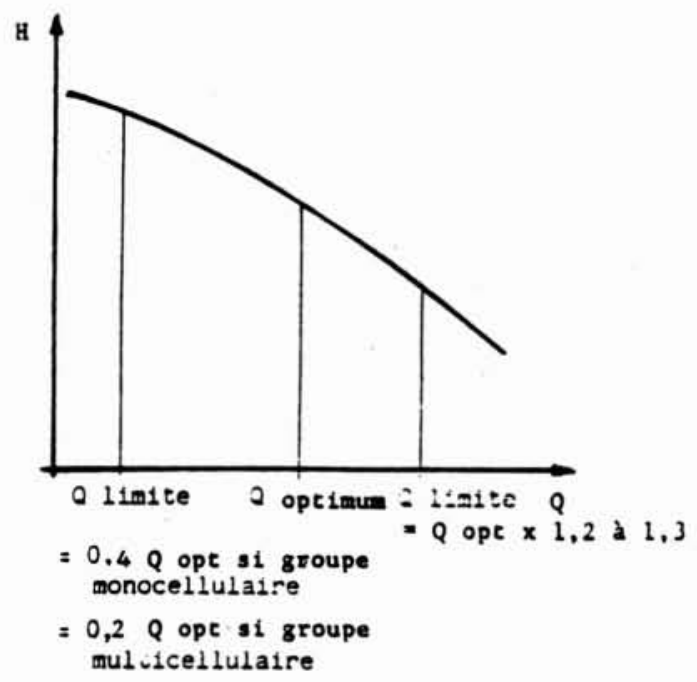

5. Pas de régulation.

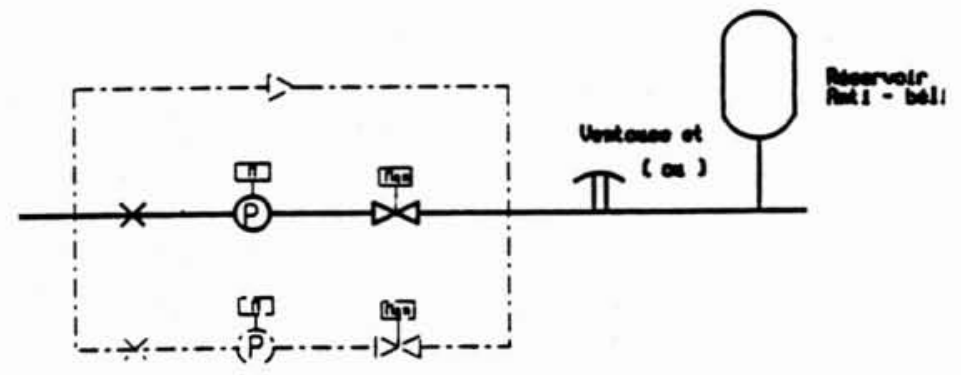

5bis. Solution 1 . 


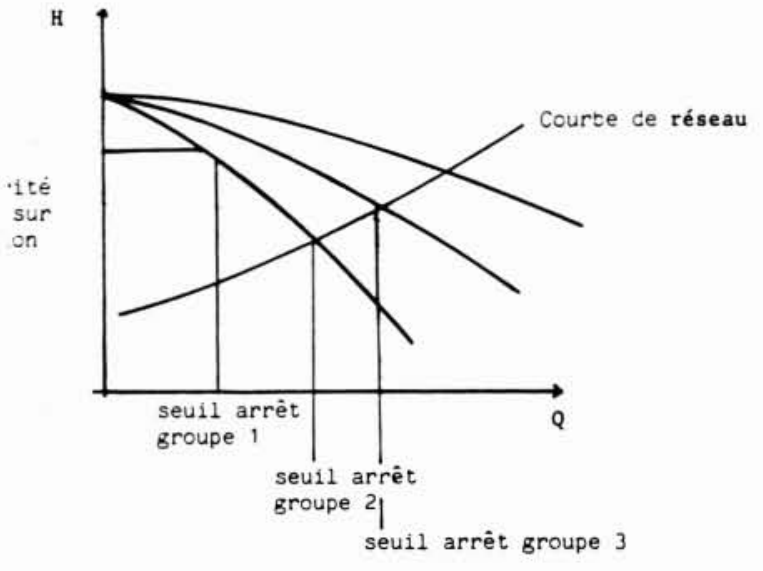

6. Arrêts sur seuils de débit.

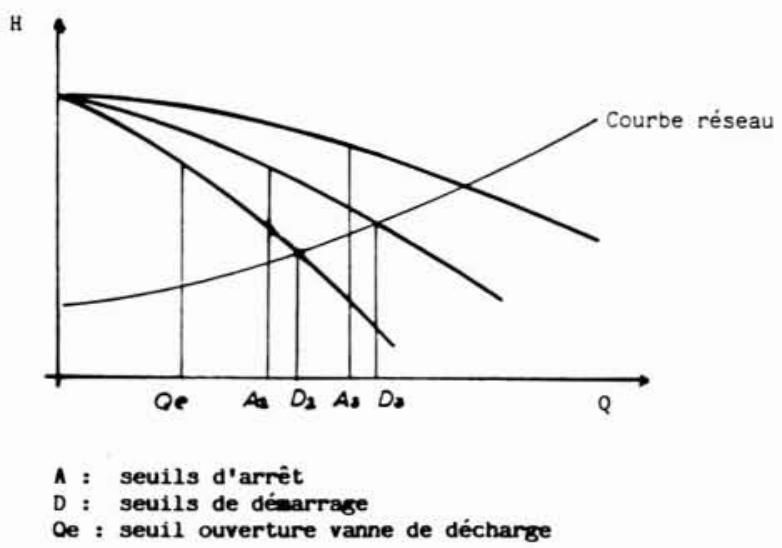

7. Régulation sur décharge.

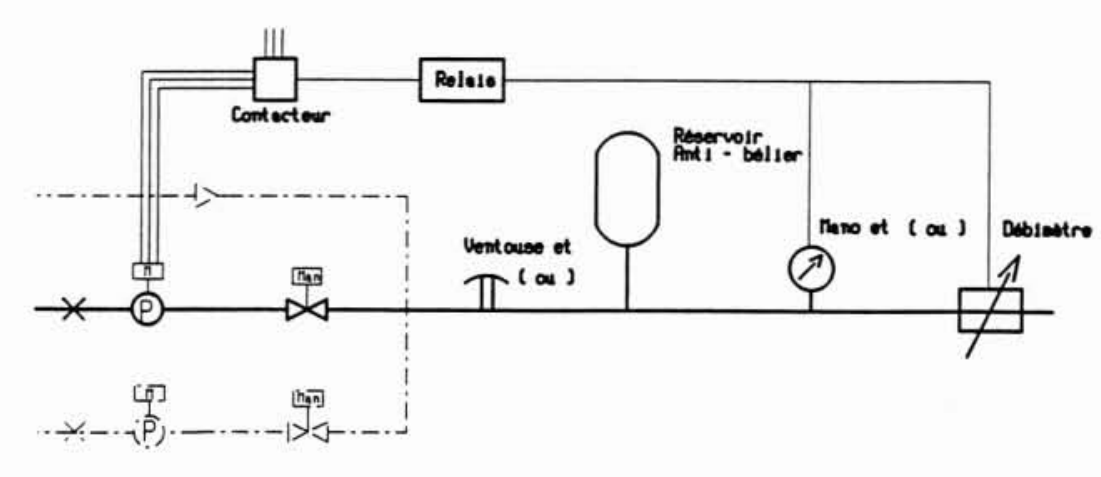

6bis. Solution 2.

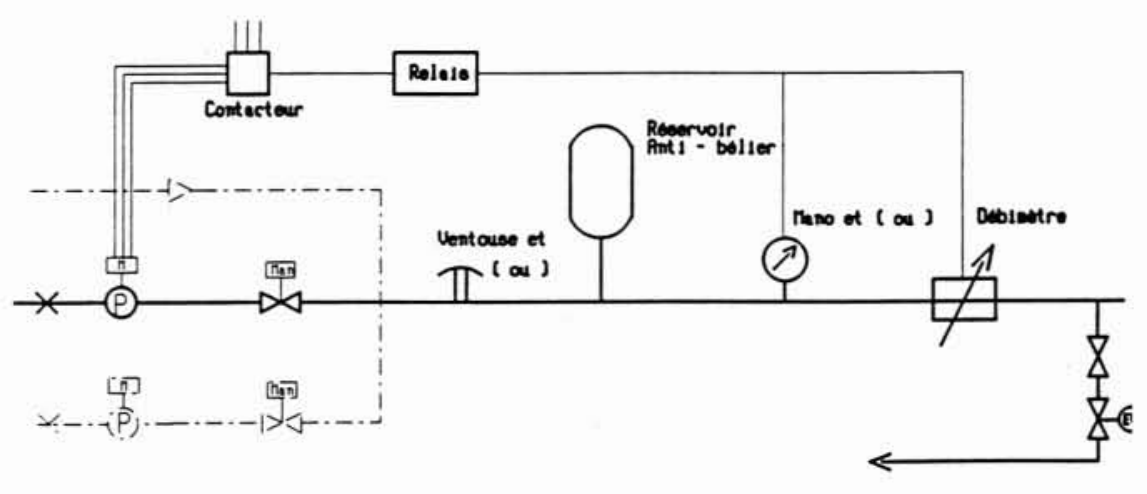

Rotour a I' eepiration

7bis. Solution 3 .

Solution 2: Arrêt automatique

Si le démarrage automatique d'une station sur un réseau d'irrigation pose toujours un problème à cause du remplissage des conduites, car on considère ici, ce qui est le cas le plus commun aujourd'hui, que l'on ne dispose pas de réserve d'eau surélevée (réservoirs, barrage, château d'eau...), l'arrêt est sans conséquence si le problème des régimes transitoires a été bien appréhendé.

Il est donc facile et confortable pour l'exploitant de prévoir un arrêt automatique, sur horloge, détection de pression haute, baisse de débit, etc...

Lorsque plusieurs groupes sont en présence, leur arrêt successif peut intervenir selon l'allure des courbes de pompe, en fonction de seuils de pression ou de débit (voir schémas 6 et 6 bis).

\section{Solution 3: Régulation sur décharge}

Dans de nombreux cas l'arrêt du prélèvement de débit peut n'être que momentané et il n'est pas nécessaire alors, d'interrompre le fonctionnement de la station. Une solution est de prévoir le retour d'une partie du débit vers l'aspiration. La fermeture de cette décharge interviendra lorsque les conditions normales seront revenues. Dans le cas de plusieurs groupes de pompage, le nombre de seuils à prévoir pour l'arrêt et le démarrage des groupes est donc double à celui du cas précédent.

Ce type de régulation permet d'éviter des investissements (volume tampon), par contre elle conduit à une majoration sensible des dépenses d'énergie en cas de fonctionnement prolongé sur décharge aux faibles débits.

On notera que dans cette solution on ne dispose d'aucun volume tampon permettant dans les valeurs seuils de débits ou de pression, de garantir une fréquence maximale de démarrage, les variations de débits doivent donc être lentes et continues. Une sécurité possible est d'arrêter un groupe qui aurait atteint sa valeur limite dans un intervalle de temps donné (voir schémas 7 et 7 bis).

Cette décharge sera calculée pour assurer un débit de sécurité à la plus petite pompe correspondant en général à :

- $40 \%$ du débit nominal pour les groupes monocellulaires ;

- 20 à $30 \%$ du débit nominal pour les groupes multicellulaires. 


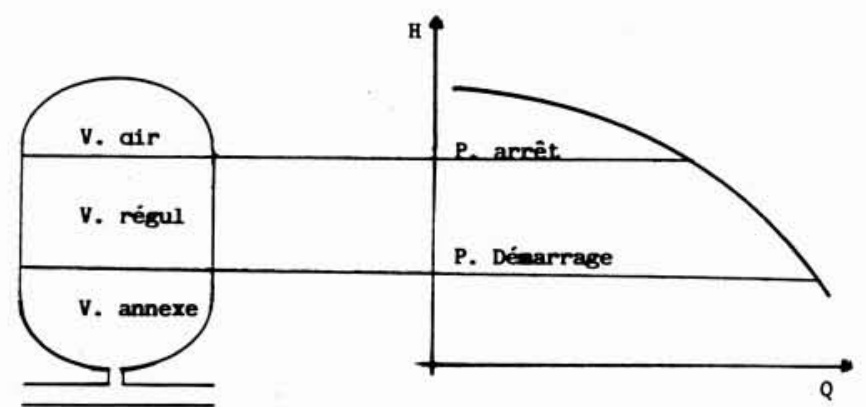

8. Calcul du volume d'un réservoir de régulation.

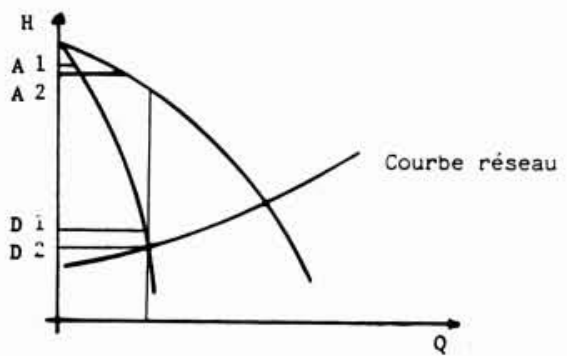

9. Régulation manométrique.

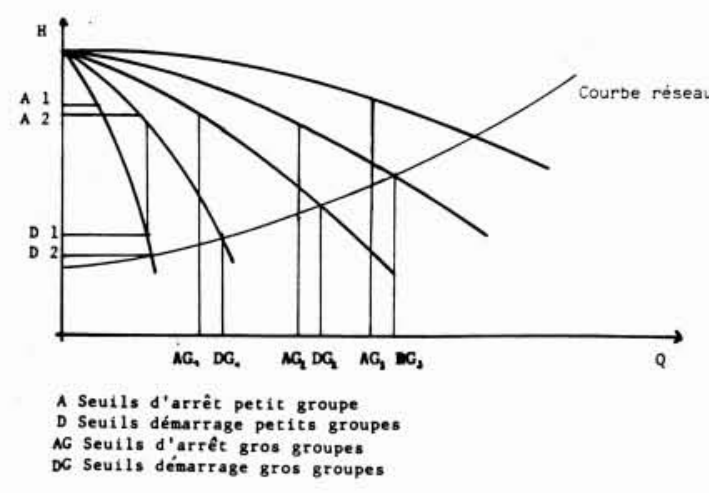

10. Régulation manodébitmétrique.

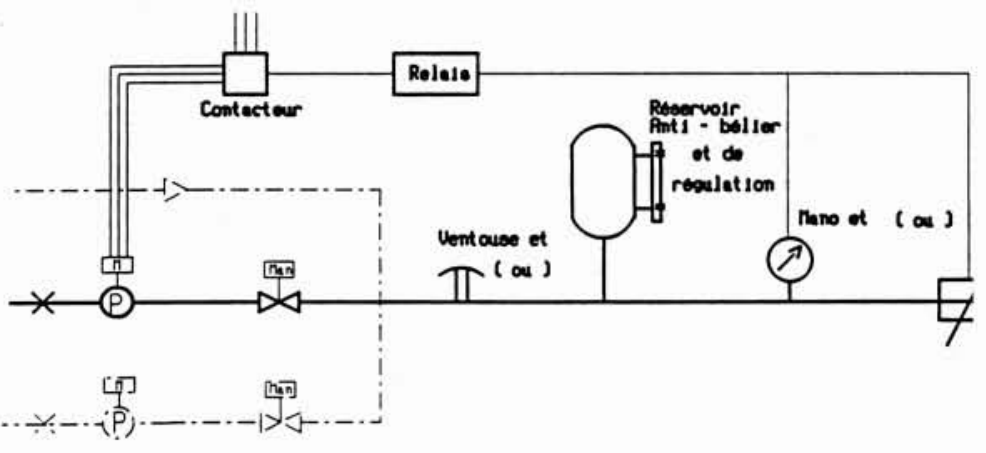

10bis. Solution 4.
Solution 4: La régulation manométrique ou manodébitmétrique

Pour éviter le risque de démarrage et d'arrêts successifs, une solution consiste à créer un volume tampon ou de régulation entre la station de pompage et le réseau. Auparavant, il était créé dans un réservoir surélevé (château d'eau) mais plus communément aujourd'hui dans un réservoir au sol sous pression.

On connaît les règles de calcul du volume d'un réservoir d'eau sous pression (voir schémas 8 et 9).

$\mathrm{V} \cdot$ total $=\mathrm{V} \cdot$ air $+\mathrm{V} \cdot$ régul $+\mathrm{V} \cdot$ annexe

$* \mathrm{~V} \cdot$ régul $=\mathrm{QT} / 4$ s'il existe la possibilité de fonctionner longtemps à un débit appelé égal à la moitié du débit de la pompe

$=\mathrm{QT} / 6$ si tous les débits sont équiprobables

$\mathrm{Q}=\mathrm{Q}$ Moyen $=\frac{2}{3}\left(\mathrm{QA}^{2}+\mathrm{QA} \times \mathrm{QB}+\mathrm{QB}^{2}\right) /(\mathrm{QA}+\mathrm{QB})$

$\mathrm{T}=$ intervalle de temps acceptable entre 2 démarrages selon les critères électriques et mécaniques des moteurs.

Il n'existe pas à proprement parler de normes sur cette valeur : à BRL, nous utilisons selon notre expérience d'exploitant :

$$
\begin{array}{lrr}
\mathrm{P}<100 \mathrm{~kW} & 4 \mathrm{dém} / \mathrm{h} & \mathrm{T}=900 \mathrm{~s} \\
100<\mathrm{P}<400 \mathrm{kw} & 2 \mathrm{dém} / \mathrm{h} & \mathrm{T}=1800 \mathrm{~s} \\
\mathrm{P}>400 \mathrm{kw} & 1 \mathrm{dém} / \mathrm{h} & \mathrm{T}=3600 \mathrm{~s} \\
* \mathrm{~V} \cdot \text { air selon Mariotte }=(\text { Vregul } \times & \mathrm{Pdem}) /(\text { Delta } \mathrm{P}) \\
* \mathrm{~V} \cdot \text { annexes selon les projets peut correspondre à : }
\end{array}
$$

— une marge de sécurité par rapport aux calculs cidessus ;

- un volume pour la protection anti-bélier à l'arrêt des groupes (il faut vérifier que le volume $\mathrm{V} \cdot \mathrm{air}+\mathrm{V} \cdot$ régul est suffisant pour la protection au démarrage des groupes); - un volume pour le maintien en pression du réseau en cas de coupure de 15 secondes sur le réseau EDF.

On sait que ces calculs amènent des volumes assez importants par exemple :

\begin{tabular}{|l|c|l|l|}
\hline \multicolumn{2}{|l|}{ Données } & \multicolumn{2}{c|}{ Résultats } \\
\hline 1 groupe & $70 \mathrm{kw}$ & VR & $11 \mathrm{~m}^{3}$ \\
P Arrêt & 110 mètres & Vair & $34 \mathrm{~m}^{3}$ \\
P Dem & 80 mètres & & \\
Qm & $50 \mathrm{l} / \mathrm{s}$ & & \\
\hline
\end{tabular}

Pour diminuer les débits moyens et donc les volumes, on est donc amené à installer des petits groupes appelés souvent "groupes jokey " assurant selon les cas mentionnés ci-dessus, 15 à $40 \%$ du débit des groupes de taille plus importante qui démarrent eux, en fonction de consignes de débit.

On parle alors de régulation mano-débitmétrique (voir schémas 10 et 10 bis). 

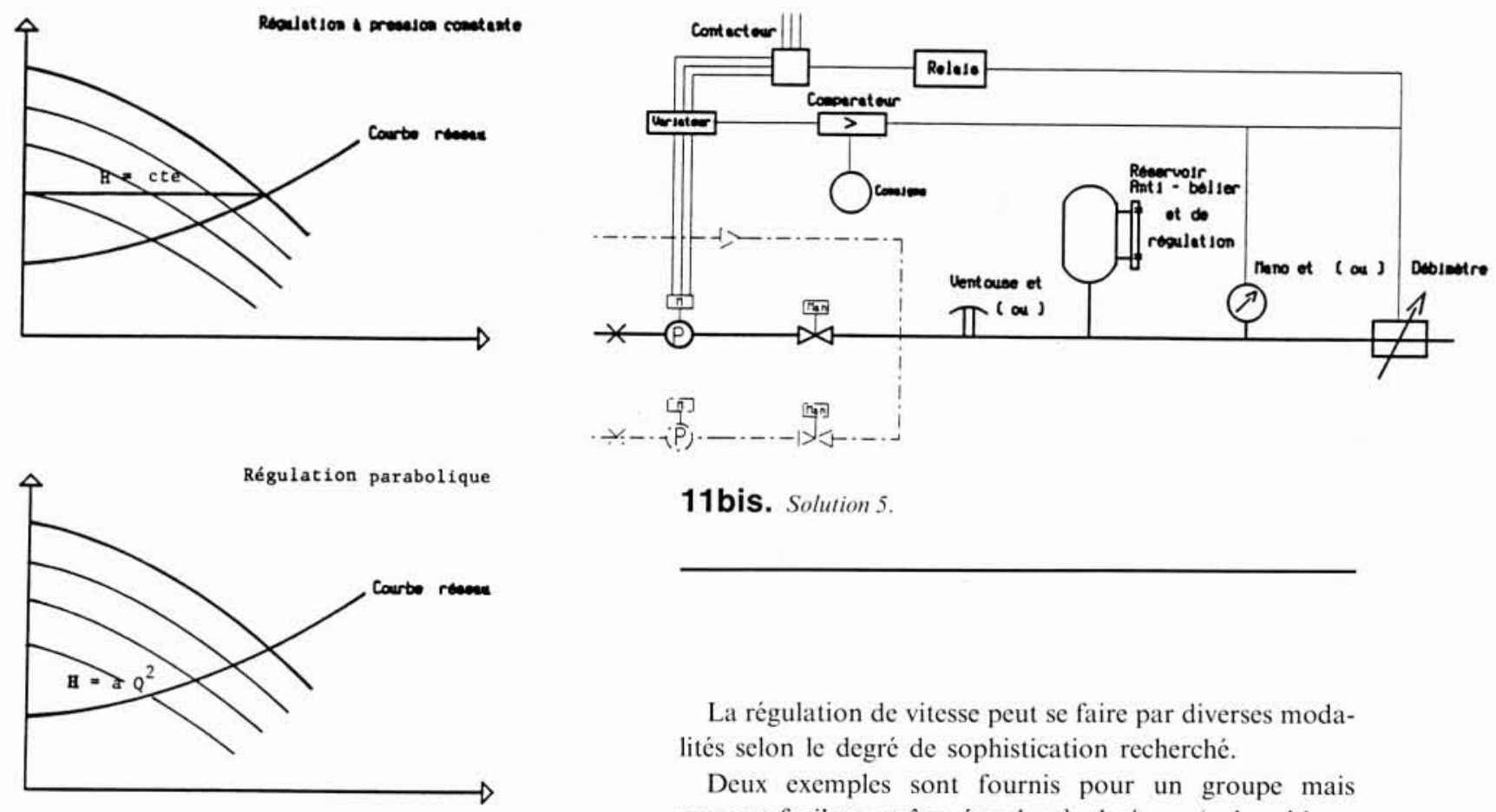

11bis. Solution 5 .

11. Régulation par vitesse variable.
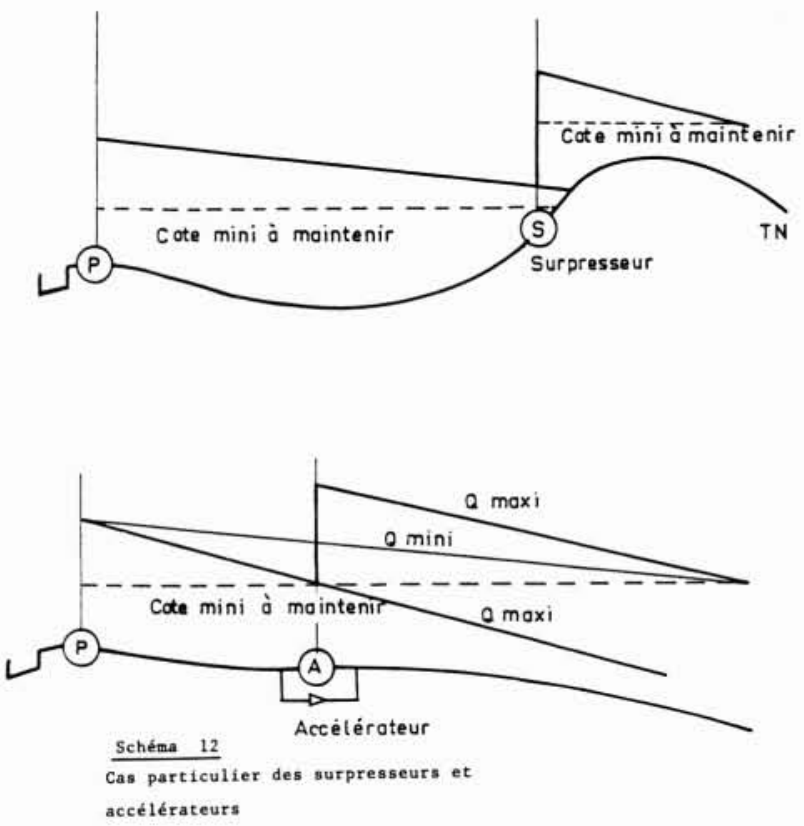

12. Cas particulier des surpresseurs et accélérateurs.

Solution 5: La régulation par vitesse variable

Le développement d'une électronique de puissance à un coût raisonnable permet dans certains cas le remplacement du réservoir d'air par un fonctionnement à vitesse variable du ou de certain(s) groupe(s) de pompage(s).

En effet, en faisant varier la vitesse en continu de façon à suivre le débit appelé par le réseau on s'affranchit de la nécessité d'une réserve tampon, pour la régulation.

On rapellera qu'après "l'ère" du moteur à courant continu, c'est maintenant le moteur asynchrone avec variation de fréquence qui s'impose pour les puissances usuelles, les cascades hypo-synchrones et les moteurs synchrones étant réservés aux puissances supérieures à $1000 \mathrm{kw}$.

La régulation de vitesse peut se faire par diverses modalités selon le degré de sophistication recherché.

Deux exemples sont fournis pour un groupe mais peuvent facilement être étendus à plusieurs (voir schémas 11 et 11 bis $)$.

Il va de soi qu'une telle régulation peut être complétée par l'un des dispositifs décrit ci-dessus comme la vanne de décharge ou un groupe jokey pour éviter des fonctionnements à trop bas débit (pour la pompe) ou trop basse vitesse (pour le moteur ou le variateur).

Comme il a été indiqué plus haut, ce dispositif permet normalement la suppression du réservoir d'air et c'est bien dans ce contexte qu'il est utilisé. Toutefois deux considérations majeures amènent souvent à le maintenir :

- la nécessité à partir d'un certain débit de disposer d'un organe de protection contre les dépressions, sûr et surtout pouvant être calculé à partir de programmes sur ordinateurs simples ;

- le contexte des appels d'offres par installation, souvent pour des stations de pompage complètes, peut amener une certaine disparité dans les équipements de variation de vitesse, d'où un manque de formation et de pièces de rechange chez un exploitant en charge de plusieurs installations. On peut alors être tenté en cas d'incident de « bypasser » le variateur pour faire fonctionner l'installation à vitesse fixe d'oủ la nécessité de maintien d'un volume de régulation.

\section{Cas particuliers des surpresseurs et des accéléra- teurs}

Le développement des réseaux et le souci de différer certains investissements amènent parfois à compléter les ouvrages de pompage de tête avec des équipements de surpression (qui fonctionnent en permanence dès la première demande de débit à l'aval ou des accélérateurs (qui sont nécessaires à partir d'un certain seuil de débit).

Le fonctionnement de tels appareils peut être alors bien spécifique (voir schéma 12). 
Le surpresseur: Sa particularité est de nécessiter de façon quasi obligatoire une protection anti-bélier par réservoir, à la fois à l'amont et à l'aval. Il est donc tentant d'utiliser ce dernier pour faire une régulation mano ou mano-débitmétrique.

Par contre on peut se trouver face à de telles variations de cote piézométrique amont et aval qu'il soit impossible de trouver une pompe pouvant fonctionner dans des conditions satisfaisantes mécanique ou énergétique; la vitesse variable est alors la seule issue.
L'accélérateur : Dans ce cas le rétablissement immédiat du débit gravitaire par le clapet permet souvent de s'affranchir de réservoirs anti-béliers.

Le démarrage ou l'arrêt du ou des groupes peut s'effectuer par mesure de débit.

Comme dans le cas précédent selon les variations de pression amont et aval, on peut être amener à utiliser des pompes à vitesse variable; il existe aussi la possibilité de mettre des groupes en série (en général 2 au maximum pour éviter les contraintes sur les pièces d'aspiration).

\section{Les critères de sélection}

Dans le tableau ci-après on a essayé de faire la liste d'un certain nombre de critères pouvant permettre d'orienter le choix vers l'une ou l'autre solution, il va de soi qu'il ne s'agit là que de points de repères individuels, la combinaison de plusieurs critères pouvant amener un conseil différent de celui obtenu avec chacun d'entre eux pris indépendamment.

\begin{tabular}{|c|c|c|c|c|c|c|}
\hline $\begin{array}{l}\text { Type } \\
\text { de } \\
\text { critères }\end{array}$ & & $\begin{array}{c}\text { Ne rien } \\
\text { faire }\end{array}$ & $\begin{array}{c}\text { Arrêt } \\
\text { automatique }\end{array}$ & $\begin{array}{l}\text { Vanne de } \\
\text { décharge }\end{array}$ & $\begin{array}{c}\text { Mano ou } \\
\text { mano } \\
\text { débitmétrique }\end{array}$ & $\begin{array}{l}\text { Vitesse } \\
\text { variable }\end{array}$ \\
\hline Exploitant & $\begin{array}{l}\text { Exploitation locale } \\
\text { non qualifiée } \\
\text { Exploitation locale } \\
\text { qualifiée } \\
\text { Exploitation } \\
\text { centralisée } \\
\text { qualifiée }\end{array}$ & $\begin{array}{l}\text { TB } \\
\text { B }\end{array}$ & $\begin{array}{l}B \\
B\end{array}$ & $\begin{array}{l}\text { B } \\
\text { TB } \\
\text { B }\end{array}$ & $\begin{array}{l}\text { TB } \\
\text { TB }\end{array}$ & $\begin{array}{l}\mathrm{M} \\
\mathrm{TB}\end{array}$ \\
\hline Débit & $\begin{array}{l}\text { Débit faible } \\
\quad<100 \mathrm{l} / \mathrm{s} \text { peu d'utilisateurs } \\
\text { Débit moyen } \\
\quad(<250 \mathrm{l} / \mathrm{s}) \\
\text { Débit important } \\
\quad(>250 \mathrm{I} / \mathrm{s})\end{array}$ & TB & TB & $\begin{array}{l}\text { TB } \\
\text { B }\end{array}$ & $\begin{array}{l}\text { B } \\
\text { TB } \\
\text { TB }\end{array}$ & $\begin{array}{l}\mathrm{B} \\
\mathrm{TB}\end{array}$ \\
\hline $\begin{array}{c}\text { Temps } \\
\text { de } \\
\text { fonctionnement }\end{array}$ & $\begin{array}{l}\text { Temps de } \\
\text { fonctionnement faible }(<1000 \mathrm{~h}) \\
\text { Temps de } \\
\text { fonctionnement moyen }(1000<3000 \mathrm{~h}) \\
\text { Temps de } \\
\text { fonctionnement fort }(>3000 \mathrm{~h})\end{array}$ & B & $\begin{array}{l}B \\
M\end{array}$ & B & $\begin{array}{l}\text { B } \\
\text { TB }\end{array}$ & $\begin{array}{l}\text { B } \\
\text { TB }\end{array}$ \\
\hline $\begin{array}{c}\text { Variation } \\
\text { de } \\
\text { débit }\end{array}$ & $\begin{array}{l}\text { Variations } \\
\text { temporelles } \\
\text { faibles } \\
\text { Variations } \\
\text { temporelles } \\
\text { fortes }\end{array}$ & TB & TB & TB & $\begin{array}{l}\text { B } \\
\text { TB }\end{array}$ & TB \\
\hline Ambiance & $\begin{array}{l}\text { Ambiance normale } \\
\text { Ambiance rude } \\
\left(\mathrm{T}^{\star}, \text { coup de foudre, humidité, }\right. \\
\text { poussière, etc...) }\end{array}$ & TB & TB & TB & TB & TB \\
\hline
\end{tabular}




\begin{tabular}{|l|c|c|c|}
\hline & Station 1 & Station 2 & Station 3 \\
\hline Débit total (I/s) & 170 & 300 & 800 \\
HMT (m) & 80 & 100 & 120 \\
Groupes de pompage & $2 \times 15 \mathrm{l} / \mathrm{s}$ & $2 \times 15$ & 30 \\
Type de pompe & $2 \times 70 \mathrm{l} / \mathrm{s}$ & $3 \times 100$ & $4 \times 200$ \\
multi cell \\
\hline
\end{tabular}

\begin{tabular}{|c|c|c|c|c|c|}
\hline $\begin{array}{c}\text { Solutions } \\
\text { Stations }\end{array}$ & 1 & 2 & 3 & 4 & $5^{*}$ \\
\hline 1 & 385 & 570 & 590 & 800 & 1160 \\
\hline 2 & 1010 & 1220 & 1240 & 1500 & 1850 \\
\hline 3 & 2390 & 2670 & 2690 & 3250 & 3550 \\
\hline
\end{tabular}

* seulement deux gros groupes à vitesse variable.

\begin{tabular}{|c|c|c|c|c|c|}
\hline $\begin{array}{c}\text { Solutions } \\
\text { Stations }\end{array}$ & 1 & 2 & 3 & 4 & 5 \\
\hline 1 & 0 & $+48 \%$ & $+53 \%$ & $+107 \%$ & $+200 \%$ \\
\hline 2 & 0 & $+20 \%$ & $+22 \%$ & $+48 \%$ & $+83 \%$ \\
\hline 3 & 0 & $+12 \%$ & $+12,5 \%$ & $+35 \%$ & $+49 \%$ \\
\hline
\end{tabular}

\section{Comparaisons économiques}

Si les critères qualitatifs sont importants, la comparaison économique entre les deux solutions ne l'est pas moins; elle peut se faire avec trois niveaux de complexité :

- la simple comparaison économique des investissements ;

- l'analyse des coûts d'énergie ;

- le calcul économique complet prenant en compte les frais de maintenance et frais financiers.

Nous traiterons successivement ces trois aspects.

\subsection{Comparaison économique}

Cette comparaison a été effectuée pour trois stations de pompage représentant assez bien l'échantillon des stations étudiées par BRL, en France et à l'étranger (tableau A).

En prenant la solution 1 comme base, on aboutit aux coûts donnés au tableau $B$ pour les 5 solutions définies ci-avant (en $10^{3}$ Francs). Le tableau $C$ donne le surcoût par rapport à la solution 1 , en valeur relative.

On voit aisément le poids important des équipements de régulation en valeur relative dans les stations de petits débits.

Si cette valeur diminue lorsque le débit augmente, le surcoût des solutions 4 et 5 reste très net en valeur absolue.
On peut toutefois noter que l'écart entre vitesse fixe et vitese variable a nettement diminué au cours de ces dix dernières années.

\subsection{Analyse des coûts d'énergie}

L'analyse des coûts d'énergie sera d'autant plus complexe mais d'autant plus fiable que l'on connaîtra les débits à fournir par mois, par jour, voire par tranches horaires chaque jour ou au moins en terme de volume annuel par tranche de débit.

On peut alors émettre un certain nombre de possibilités de régulation d'un type unique ou combinées, pour un débit pouvant varier de 0 à $Q \max$.

En irrigation sous pression, les variations de débit dans l'année sont importantes et les solutions nombreuses ; aussi avons-nous été amenés à réaliser un logiciel de calcul dont les données de base sont:

- les courbes de pompes,

- le mode de régulation par tranches de débit par exemple :

de 0 à Q1 manométrique,

$\mathrm{Q} 1$ à $\mathrm{Q} 2$ vitesse fixe,

$\mathrm{Q} 2$ à $\mathrm{Q} 3$ vitesse variable $\mathrm{H}=$ cte,

$\mathrm{Q} 3$ à $\mathrm{Q} 4$ vitesse variable $\mathrm{H}=\mathrm{aQ}^{2}$.

Le résultat est la consommation d'énergie annuelle. 
13.

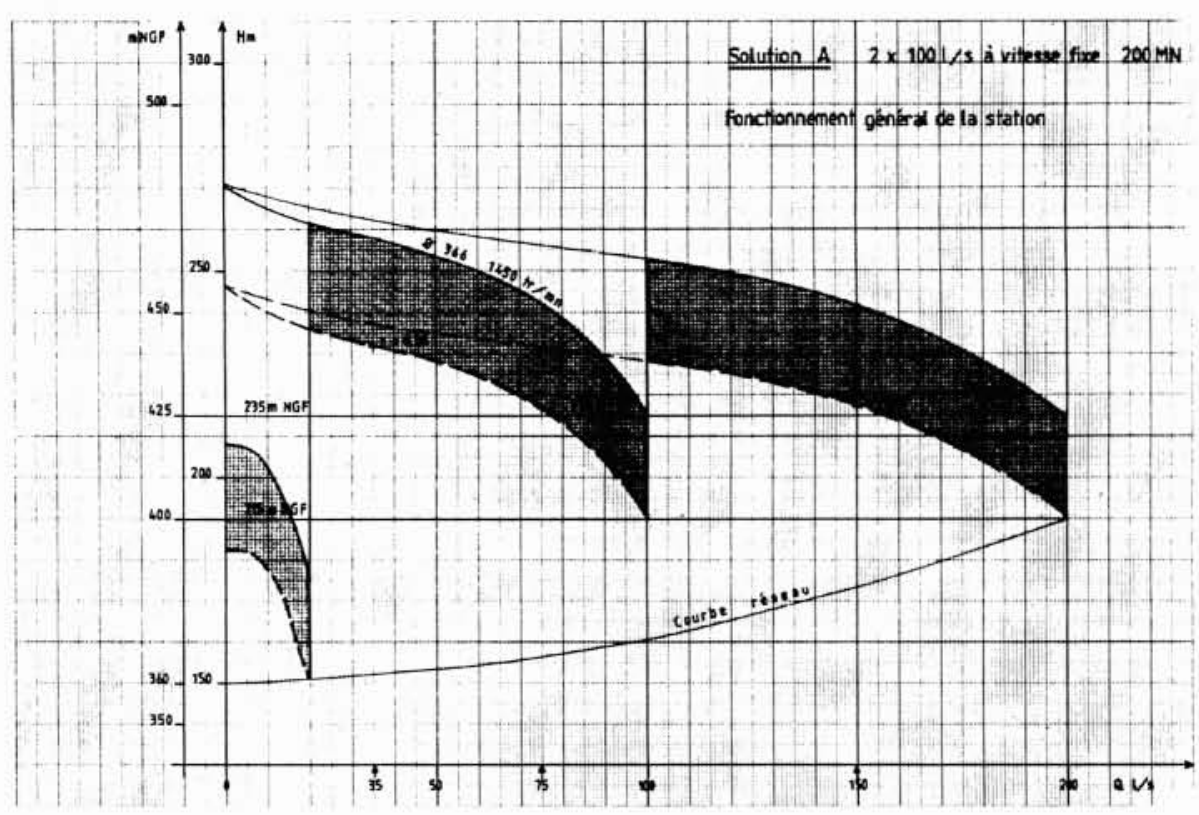

14.
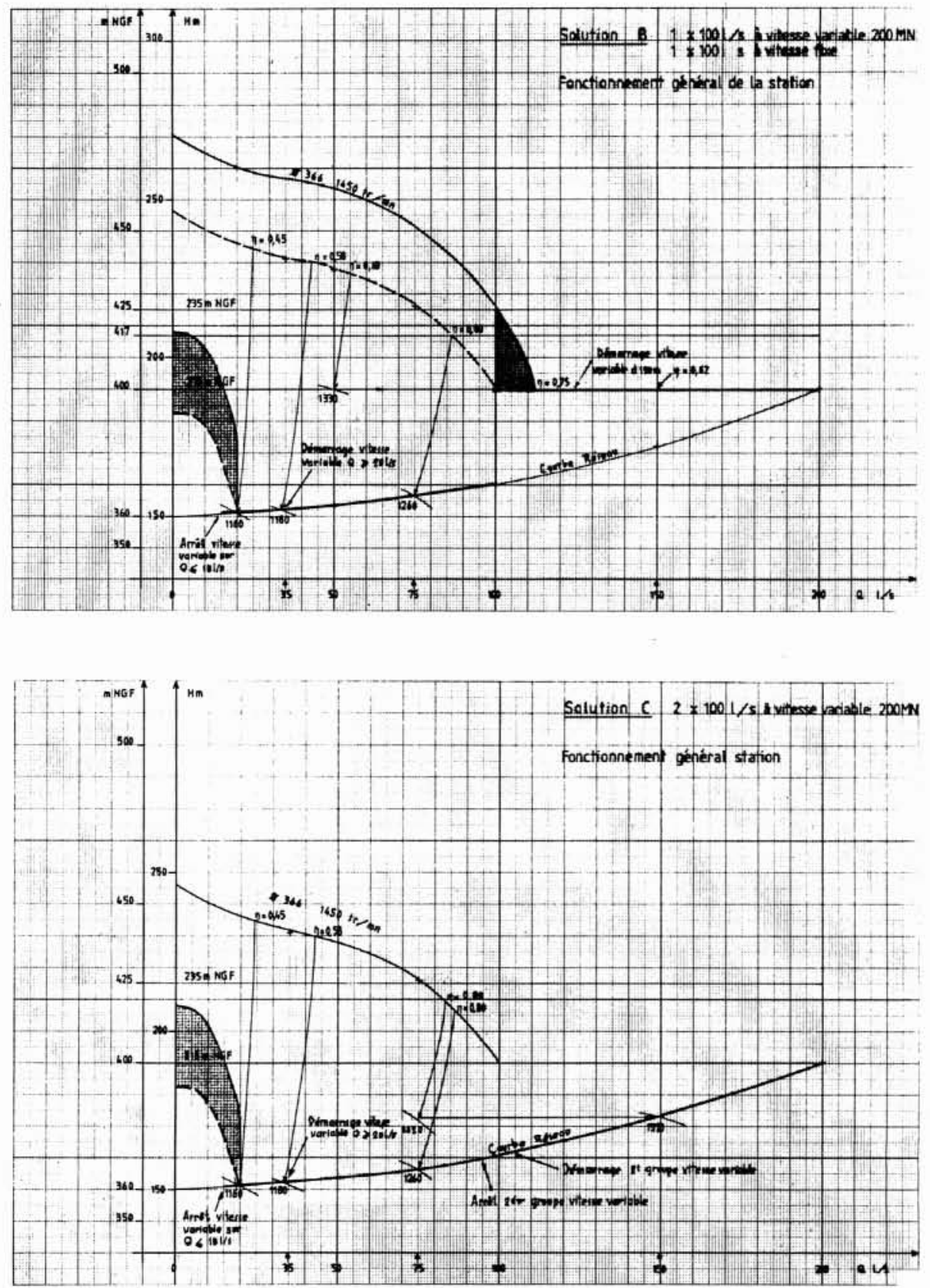
On peut noter que ce programme peut être utilisé au niveau projet mais son application peut être étendue au niveau du jugement d'offres pour la comparaison entre 2 fournisseurs de pompe.

Il n'est pas possible de donner des chiffres comparatifs entre les cinq solutions en ce qui concerne les coûts d'énergie car ceux-ci sont trop variables selon les conditions de fonctionnement mais notre expérience fixe à peu près à 2000-3000 heures le seuil de rentabilité pour l'utilisation de la vitesse variable, or en irrigation ce temps ne dépasse que très exceptionnellement 2000 heures.

\subsection{Calcul économique complet}

Le logiciel permettant ce calcul est en cours de réalisation à BRL et permettra la prise en compte :

- des investissements et de leur aspect échelonné éventuel,

- des coûts d'énergie venant du logiciel décrit ci-dessus, - des frais de maintenance et de renouvellement (bien évidemment plus élevé pour la vitesse variable).

Il en ressort le coût actualisé des scénarii de régulation projetés.

Lorsque ces études ont été réalisées à BRL leur conclusion, pour la seule irrigation, rejoint celle émise au souschapitre précédent, elles privilégient en général les solutions à vitesse fixe. On peut voir les types de solutions étudiées pour une station dans les schémas 13 à 16 .

\section{Conclusion}

La multiplicité des solutions utilisables en irrigation sous pression doit amener à une certaine prudence dans les choix. Avant tout, il importe de bien connaître les conditions de fonctionnement présentes et futures et le temps prévisible pour atteindre le régime de croisière du périmètre.

Entre une solution trop rustique qui entraînerait des dépenses de personnel exhorbitantes, une usure prématurée des équipements et une solution trop sophistiquée, coûteuse en investissement, mal adaptée aux capacités techniques de l'exploitation, il existe des possibilités intermédiaires pouvant être mises en place immédiatement ou différées dans le temps.

En tout cas lorsque ce sera possible toutes les dispositions hydrauliques ou de génie civil devront être prises au niveau des travaux pour permettre des compléments d'équipements ultérieurs (débitmètre, variateurs, etc...).

Des logiciels comme celui mis au point pour notre usage, aident à la décision pour les équipements et sont une sécurité pour le maître d'ouvrage et l'exploitant.

16.

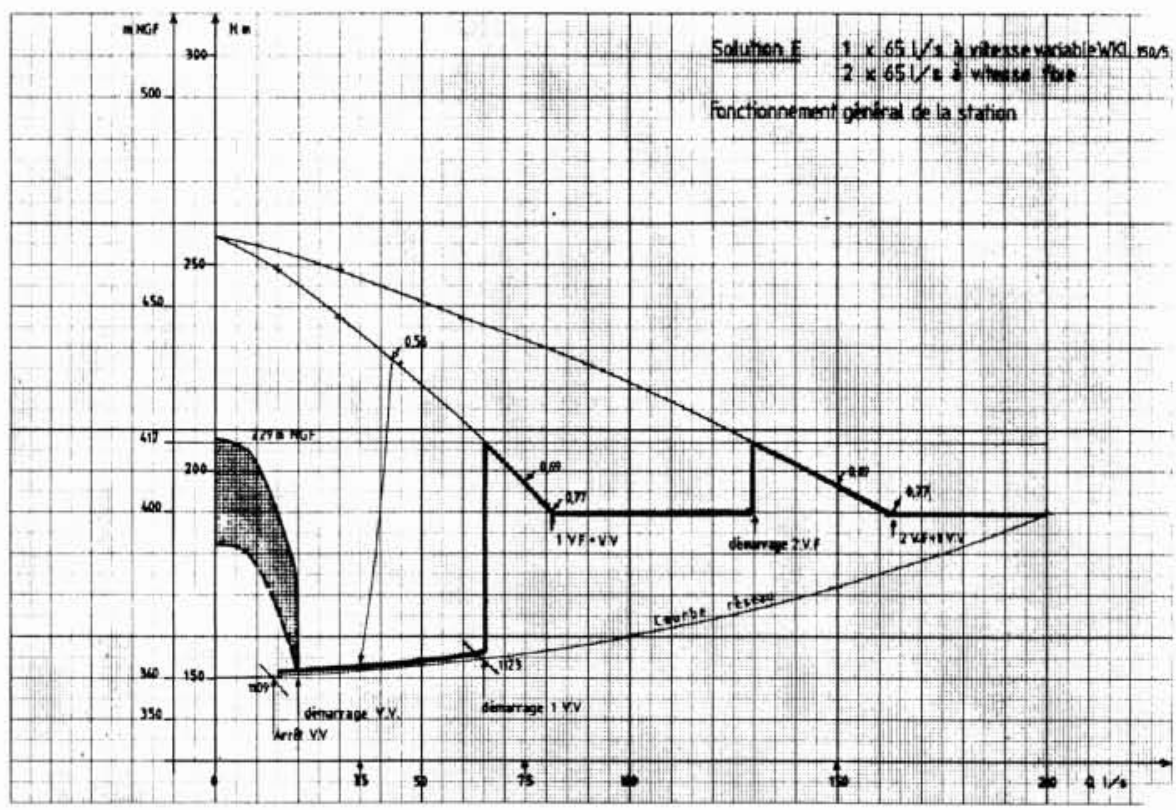




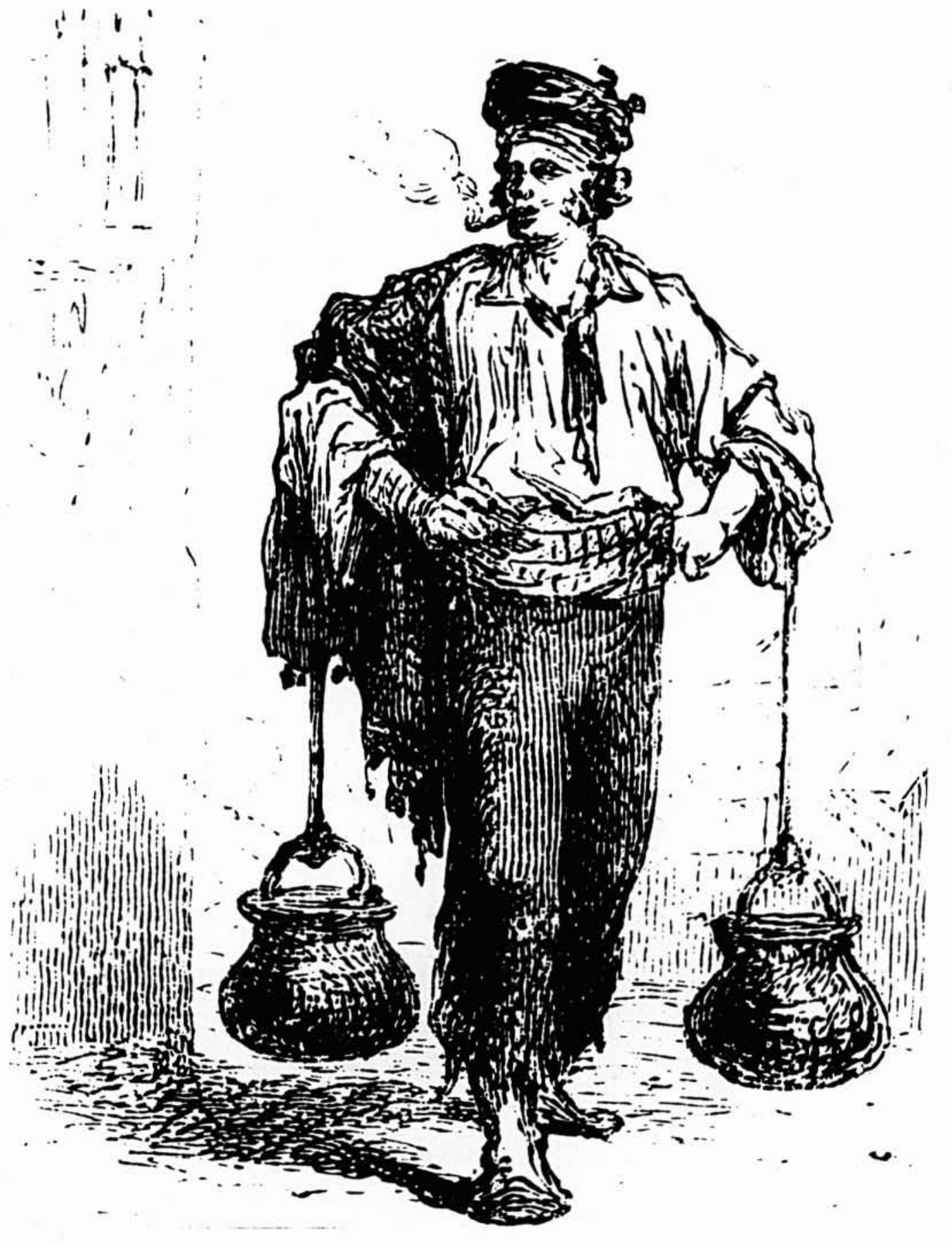

